PROCEEDINGS OF THE

AMERICAN MATHEMATICAL SOCIETY

Volume 138, Number 7, July 2010, Pages 2403-2412

S 0002-9939(10)10269-X

Article electronically published on February 24, 2010

\title{
DISCONTINUITY OF THE LEMPERT FUNCTION OF THE SPECTRAL BALL
}

\author{
PASCAL J. THOMAS AND NGUYEN VAN TRAO
}

(Communicated by Franc Forstneric)

\begin{abstract}
We give some further criteria for continuity or discontinuity of the Lempert function of the spectral ball $\Omega_{n}$, with respect to one or both of its arguments, in terms of cyclicity of the matrices involved.
\end{abstract}

\section{INTRODUCTION AND STATEMENT OF RESULTS}

The spectral ball is the set of all $n \times n$ complex matrices with eigenvalues strictly smaller than one in modulus. It can be seen as the union of all the unit balls of the space of matrices endowed with all the possible operator norms arising from a choice of norm on the space $\mathbb{C}^{n}$. It contains many entire curves. As analogues of the Montel theorem cannot hold, several invariant objects in complex analysis exhibit discontinuity phenomena in this setting; this was first pointed out in [1].

The goal of this paper is to give a few facts about discontinuities of the Lempert function, corresponding to the two-point Pick-Nevanlinna problem (compare [11).

We fix some notation. Let $\mathcal{M}_{n}$ be the set of all $n \times n$ complex matrices. For $A \in \mathcal{M}_{n}$ denote by $\operatorname{sp}(A)$ and $r(A)=\max _{\lambda \in s p(A)}|\lambda|$ the spectrum and the spectral radius of $A$, respectively. The spectral ball $\Omega_{n}$ is the set

$$
\Omega_{n}=\left\{A \in \mathcal{M}_{n}: r(A)<1\right\} .
$$

The characteristic polynomial of the matrix $A$ is

$$
P_{A}(t):=\operatorname{det}(t I-A)=: t^{n}+\sum_{j=1}^{n}(-1)^{j} \sigma_{j}(A) t^{n-j},
$$

where $I \in \mathcal{M}_{n}$ is the unit matrix. We define a holomorphic map $\sigma$ from $\mathcal{M}_{n}$ to $\mathbb{C}^{n}$ by $\sigma:=\left(\sigma_{1}, \ldots, \sigma_{n}\right)$. The symmetrized polydisk $\mathbb{G}_{n}:=\sigma\left(\Omega_{n}\right)$ is a bounded domain in $\mathbb{C}^{n}$, which is hyperconvex [6] and, therefore, a complete hyperbolic domain, and taut. As is noted in that paper or in [11, Proposition 7], $\sigma(A)=\sigma(B)$ if and only if there is an entire curve contained in $\Omega_{n}$ going through $A$ and $B$.

For general facts about invariant (pseudo)distances and (pseudo)metrics, see for instance [8]. The Lempert function of a domain $D \subset \mathbb{C}^{m}$ is defined, for $z, w \in D$, as

$$
l_{D}(z, w):=\inf \{|\alpha|: \alpha \in \mathbb{D} \text { and } \exists \varphi \in \mathcal{O}(\mathbb{D}, D): \varphi(0)=z, \varphi(\alpha)=w\} .
$$

Received by the editors March 6, 2009, and, in revised form, September 5, 2009.

2010 Mathematics Subject Classification. Primary 32F45; Secondary 32U35.

The initial version of this paper was written during the second author's stay at the Paul Sabatier University, Toulouse, with the help of the Formath Vietnam program.

(C)2010 American Mathematical Society Reverts to public domain 28 years from publication 2403 
In the unit disk $\mathbb{D}, l_{\mathbb{D}}(\lambda, \mu)=\left|\frac{\mu-\lambda}{1-\bar{\lambda} \mu}\right|$. The Lempert function is upper semicontinuous and decreases under holomorphic maps, so for $A, B \in \Omega_{n}$,

$$
l_{\Omega_{n}}(A, B) \geq l_{\mathbb{G}_{n}}(\sigma(A), \sigma(B)) .
$$

On $\mathbb{G}_{n}$, the Lempert function is continuous, and the remark above about entire curves shows that $l_{\mathbb{G}_{n}}(\sigma(A), \sigma(B))=0$ if and only if $l_{\Omega_{n}}(A, B)=0$.

For a Zariski dense open set of matrices, equality holds in (1.1) and the Lempert function is continuous. Recall that a matrix $A$ is cyclic (or non-derogatory) if it admits a cyclic vector (see for instance [7]). As in [11, we denote by $C_{\sigma(A)}$ the companion matrix of the characteristic polynomial of $A ; A$ is cyclic if and only if it is conjugate to $C_{\sigma(A)}$.

Agler and Young [1] proved that if $A$ and $B$ are cyclic, any holomorphic mapping $\varphi \in \mathcal{O}\left(\mathbb{D}, \mathbb{G}_{n}\right)$ through $\sigma(A)$ and $\sigma(B)$ lifts to $\Phi \in \mathcal{O}\left(\mathbb{D}, \Omega_{n}\right)$ through $A$ and $B$, so that in particular:

Proposition 1.1 (Agler-Young). If $A, B \in \Omega_{n}$ are cyclic, then

$$
l_{\Omega_{n}}(A, B)=l_{\mathbb{G}_{n}}(\sigma(A), \sigma(B)) .
$$

Continuity of the Lempert function near such a pair $(A, B)$ follows from the fact that cyclicity is an open condition or from the following.

Proposition 1.2. Let $A, B \in \Omega_{n}$.

(1) The Lempert function $l_{\Omega_{n}}$ is continuous at $(A, B)$ if and only if (1.2) holds.

(2) If $B$ is cyclic and the function $l_{\Omega_{n}}(., B)$ is continuous at $A$, then (1.2) holds.

The proofs are given in Section 2

We see that when $B$ is cyclic, continuity of the Lempert function with respect to both variables reduces to continuity with respect to the first variable. We now study this partial continuity for itself.

Theorem 1.3. Let $A \in \Omega_{n}$. If $A$ is not cyclic (or derogatory), then there exists a matrix $B \in \Omega_{n}$ such that the function $l_{\Omega_{n}}(., B)$ is not continuous at $A$.

The proof is given in Section 3 ,

Conversely, suppose that $A$ is cyclic. If $B$ is cyclic, then Proposition 1.1 settles the question. If not, the converse holds in several interesting cases.

Proposition 1.4. Let $A, B \in \Omega_{n}$, A cyclic. If $B$ has only one eigenvalue, or $n \leq 3$, then the function $l_{\Omega_{n}}(., B)$ is continuous at $A$.

This is proved in Section 4

We conjecture that the additional hypotheses on $B$ or $n$ can be dispensed with. When $A$ is derogatory, one may wonder which matrices $B$ make the function $l_{\Omega_{n}}(., B)$ continuous at $A$. The case where $A=t I$, treated in [11, suggests that discontinuity is generic. We give a relevant example in Section 5. In Section 6 , we apply our results to compare the Lempert and Green functions on the spectral ball.

\section{Proof of Proposition 1.2}

2.1. Proof of (i), direct part. Since the cyclic matrices are dense in $\Omega_{n}$, there exist sequences of cyclic matrices $A_{j}, B_{j}$ such that $A_{j} \rightarrow A, B_{j} \rightarrow B$. By continuity of $l_{\Omega_{n}}$ at $(A, B)$ we get that $l_{\Omega_{n}}\left(A_{j}, B_{j}\right) \stackrel{j \rightarrow \infty}{\longrightarrow} l_{\Omega_{n}}(A, B)$. 
On the other hand, $l_{\Omega_{n}}\left(A_{j}, B_{j}\right)=l_{\mathbb{G}_{n}}\left(\sigma\left(A_{j}\right), \sigma\left(B_{j}\right)\right)$. By tautness of the domain $\mathbb{G}_{n}$ we have $l_{\mathbb{G}_{n}}\left(\sigma\left(A_{j}\right), \sigma\left(B_{j}\right)\right) \stackrel{j \rightarrow \infty}{\longrightarrow} l_{\mathbb{G}_{n}}(\sigma(A), \sigma(B))$. This implies that $l_{\Omega_{n}}(A, B)=l_{\mathbb{G}_{n}}(\sigma(A), \sigma(B))$.

2.2. Proof of (i), converse part. Assume that $l_{\Omega_{n}}(A, B)=l_{\mathbb{G}_{n}}(\sigma(A), \sigma(B))$.

Let $\left(A_{j}, B_{j}\right) \subset \Omega_{n}$ be such that $\left(A_{j}, B_{j}\right) \stackrel{j \rightarrow \infty}{\longrightarrow}(A, B)$ and

$$
\lim _{j \rightarrow \infty} l_{\Omega_{n}}\left(A_{j}, B_{j}\right)=a:=\liminf _{(X, Y) \rightarrow(A, B)} l_{\Omega_{n}}(X, Y) .
$$

We have

$$
l_{\Omega_{n}}\left(A_{j}, B_{j}\right) \geq l_{\mathbb{G}_{n}}\left(\sigma\left(A_{j}\right), \sigma\left(B_{j}\right)\right) \rightarrow l_{\mathbb{G}_{n}}(\sigma(A), \sigma(B)),
$$

and hence $a \geq l_{\mathbb{G}_{n}}(\sigma(A), \sigma(B))=l_{\Omega_{n}}(A, B)$. Then $l_{\Omega_{n}}$ is lower semicontinuous at $(A, B)$. Since $l_{\Omega_{n}}$ is always upper semicontinous, it is continuous at $(A, B)$.

2.3. Proof of (ii). We only need to repeat the proof of the direct part of (i), taking $B_{j}=B$ for all $j$. Then we use the continuity of $l_{\Omega_{n}}$ in the first variable.

\section{Proof of Theorem 1.3}

We shall need a theorem by Bharali [3]. If $\lambda \in s p(W)$, then let $m(\lambda)$ denote the multiplicity of $\lambda$ as a zero of the minimal polynomial of $W$.

Theorem 3.1 (Bharali). Let $F \in \mathcal{O}\left(\mathbb{D}, \Omega_{n}\right), n \geq 2$, and let $\zeta_{1}, \zeta_{2} \in \mathbb{D}$. Write $W_{j}=F\left(\zeta_{j}\right), j=1,2$. Then

$$
\begin{aligned}
\max \left\{\max _{\mu \in s p\left(W_{2}\right)} \prod_{\lambda \in s p\left(W_{1}\right)} l_{\mathbb{D}}(\lambda, \mu)^{m(\lambda)} ; \max _{\lambda \in s p\left(W_{1}\right)} \prod_{\mu \in s p\left(W_{2}\right)} l_{\mathbb{D}}(\lambda, \mu)^{m(\mu)}\right\} & \\
& \leq l_{\mathbb{D}}\left(\zeta_{1}, \zeta_{2}\right) .
\end{aligned}
$$

Now, let $A$ be derogatory. The idea will be to construct a matrix $B$ a short distance away from $A$ into a direction which belongs to the kernel of the differential map of $\sigma$ at $A$, but where the Kobayashi-Royden pseudometric does not vanish. Compare this with the proof of Proposition 3 and in particular Lemma 8 in [10].

Since $A$ is derogatory, at least two of the eigenvalues of $A$ are equal, say to $\lambda$. Applying the automorphism of $\Omega_{n}$ given by $M \mapsto(\lambda I-M)(I-\bar{\lambda} M)^{-1}$, we may assume that $\lambda=0$. Since the map $A \rightarrow P^{-1} A P$ is a linear automorphism of $\Omega_{n}$ for any $P \in \mathcal{M}_{n}^{-1}$, we may also assume that $A$ is in Jordan form. In particular,

$$
A=\left(\begin{array}{cc}
A_{0} & 0 \\
0 & A_{1}
\end{array}\right)
$$

where $A_{0} \in \mathcal{M}_{m}, 2 \leq m \leq n, s p\left(A_{0}\right)=\{0\}, A_{1} \in \mathcal{M}_{n-m}, 0 \notin s p\left(A_{1}\right)$. Furthermore, there is a set $J \subsetneq\{2, \ldots, m\}$, possibly empty, such that $a_{j-1, j}=1$ for $j \in J$, and all other coefficients $a_{i, j}=0$ for $1 \leq i, j \leq m$. Denote $0 \leq r:=\# J=$ $\operatorname{rank} A_{0} \leq m-2$ and $k$ is the multiplicity of 0 as a zero of the minimal polynomial of $A_{0}, 0<k \leq r+1<m$.

We set

$$
X:=\left(\begin{array}{cc}
X_{0} & 0 \\
0 & 0
\end{array}\right) \in \mathcal{M}_{n}
$$

where $X_{0}=\left(x_{i, j}\right)_{1 \leq i, j \leq m}$ is such that $x_{j-1, j}=1$ for $j \in\{2, \ldots, m\} \backslash J, x_{m, 1}=1$, and $x_{i, j}=0$ otherwise. 
For $\delta>0$ small enough we set

$$
B=A+\delta X=\left(\begin{array}{cc}
B_{0} & 0 \\
0 & A_{1}
\end{array}\right)
$$

where $B_{0}=A_{0}+\delta X_{0}$.

We begin by computing $\sigma_{j}\left(B_{0}\right), 1 \leq j \leq m$. Expanding with respect to the first column, we see that

$$
\operatorname{det}\left(t I-B_{0}\right)=t^{m}-\delta^{m-r}
$$

The $m$ distinct roots $\lambda_{1}, \lambda_{2}, \cdots, \lambda_{m}$ of this polynomial are the eigenvalues of $B_{0}$, and the multiplicity of $\lambda_{j}$ as a zero of the minimal polynomial of $B_{0}$ is $1,1 \leq j \leq m$. Comparing the respective coefficients of both sides, it follows that

$$
\sigma_{j}\left(B_{0}\right)=\left\{\begin{array}{ll}
0, & 1 \leq j \leq m-1 \\
(-1)^{m} \delta^{m-r}, & j=m
\end{array} .\right.
$$

Let $s p\left(A_{1}\right)=\left\{\mu_{1}, \mu_{2}, \cdots, \mu_{s}\right\}$. Denote the multiplicity of $\mu_{j}$ as a zero of the minimal polynomial of $A_{1}$ as $m_{j}, 1 \leq j \leq s$.

Now consider $\varphi \in \mathcal{O}\left(\mathbb{D}, \Omega_{n}\right)$ and $\zeta \in \mathbb{D}$ such that $\varphi(0)=A, \varphi(\zeta)=B$. Then, by applying (3.1), we obtain that

$$
|\zeta| \geq \max \left\{\left|\lambda_{1} \cdots \lambda_{m} \mu_{1}^{m_{1}} \cdots \mu_{s}^{m_{s}}\right| ;\left|\lambda_{j}\right|^{k} \prod_{i=1}^{s} l_{\mathbb{D}}\left(\lambda_{j}, \mu_{i}\right)^{m_{i}}, 1 \leq j \leq m\right\} .
$$

Using this and (3.2) with $\delta$ small enough we have

$$
l_{\Omega_{n}}(A, B) \geq C \cdot \delta^{\frac{m-r}{m} k}, \text { where } C \text { is a constant. }
$$

Take a sequence of cyclic matrices $A_{0}^{j} \rightarrow A_{0}$. If we consider the matrices

$$
A^{j}:=\left(\begin{array}{cc}
A_{0}^{j} & 0 \\
0 & A_{1}
\end{array}\right) \in \Omega_{n},
$$

then $A^{j} \rightarrow A$ as $j \rightarrow \infty$.

Define the map $f: \Omega_{m} \rightarrow \Omega_{n}$ by

$$
f(M)=\left(\begin{array}{cc}
M & 0 \\
0 & A_{1}
\end{array}\right) .
$$

Since $f\left(A_{0}^{j}\right)=A^{j} ; f\left(B_{0}\right)=B$, we have

$$
l_{\Omega_{n}}\left(A^{j}, B\right) \leq l_{\Omega_{m}}\left(A_{0}^{j}, B_{0}\right) .
$$

Since $A_{0}^{j}, B_{0}$ are cyclic, we have

$$
l_{\Omega_{m}}\left(A_{0}^{j}, B_{0}\right)=l_{\mathbb{G}_{m}}\left(\sigma\left(A_{0}^{j}\right), \sigma\left(B_{0}\right)\right) .
$$

Since $\mathbb{G}_{m}$ is a taut domain, $l_{\mathbb{G}_{m}}$ is a continuous function. Thus

$$
l_{\mathbb{G}_{m}}\left(\sigma\left(A_{0}^{j}\right), \sigma\left(B_{0}\right)\right) \rightarrow l_{\mathbb{G}_{m}}\left(\sigma\left(A_{0}\right), \sigma\left(B_{0}\right)\right) .
$$

On the other hand, we can find $R>0$ such that $\mathbb{B}(0, R) \subset \mathbb{G}_{m}$, where $\mathbb{B}(0, R)$ denotes the Euclidean ball with center at 0 and radius $R$. For $\delta$ chosen small 
enough, $\sigma\left(B_{0}\right) \in \mathbb{B}(0, R)$. By the definition of the Lempert function and $[8$, Proposition 3.1.10], we conclude that

$$
\begin{aligned}
l_{\mathbb{G}_{m}}\left(\sigma\left(A_{0}\right), \sigma\left(B_{0}\right)\right) \leq l_{\mathbb{B}(0, R)} & \left(\sigma\left(A_{0}\right), \sigma\left(B_{0}\right)\right) \\
& =l_{\mathbb{B}(0, R)}\left((0, \cdots, 0),\left(0, \cdots, \delta^{m-r}\right)\right)=\frac{\delta^{m-r}}{R} .
\end{aligned}
$$

Combining (3.4), (3.5), (3.6), (3.7) and (3.8), we have

$$
l_{\Omega_{n}}(A, B)>l_{\Omega_{n}}\left(A^{j}, B\right)
$$

when $\delta$ is small enough and $j$ is sufficiently large. It implies the discontinuity of the Lempert function $l_{\Omega_{n}}(., B)$ at the point $A$.

Note that we have proved a slightly stronger statement than the proposition that for $A$ to be cyclic, it is enough that the function $l_{\Omega_{n}}(., B)$ be continuous at $A$ for all $B$ in some neighborhood of $A$.

\section{Proof of Proposition 1.4}

4.1. Lifting maps. We need the following generalization of [1, Theorem 2.8].

Proposition 4.1. Let $B \in \mathcal{M}_{n}(\mathbb{C})$ be a nilpotent matrix. Then there exists a linear map $\Theta_{B}$ from the set of analytic maps from $\mathbb{D}$ to $\mathbb{C}^{n}$, depending only on the values at 0 of the first $n-1$ derivatives of the coordinates of the map, such that:

Given $\zeta_{0} \in \mathbb{D}, A \in \Omega_{n}$ a cyclic matrix, and $\varphi$ a holomorphic map from $\mathbb{D}$ to $\mathbb{G}_{n}$ such that $\varphi(0)=\sigma(B), \varphi\left(\zeta_{0}\right)=\sigma(A)$, then there exists $\tilde{\varphi}$, a holomorphic map from $\mathbb{D}$ to $\Omega_{n}$ such that $\sigma \circ \tilde{\varphi}=\varphi, \tilde{\varphi}(0)=B$ and $\tilde{\varphi}\left(\zeta_{0}\right)=A$ if and only if $\Theta_{B}(\varphi)=0$.

Notice that in the case where $B$ is cyclic, $\sigma$ is of maximal rank at $B$, so that $\Theta_{B}=0$; in that case it was known 1 that any map $\varphi$ admits a lifting through cyclic matrices.

Finally, the case where $B$ admits a single (arbitrary) eigenvalue easily reduces to the nilpotent case by using the automorphism $\Phi_{\lambda}(M)=\left(\lambda I_{n}-M\right)\left(I_{n}-\bar{\lambda} M\right)^{-1}$, of the spectral ball.

Proof of Proposition 1.4 in the case of a single eigenvalue. It is enough to prove that $\ell:=\liminf _{M \rightarrow A} l_{\Omega_{n}}(M, B) \geq l_{\Omega_{n}}(A, B)$. Suppose that $A_{j} \rightarrow A(j \geq 1)$ and that $\tilde{\varphi}_{j} \in \mathcal{O}\left(\mathbb{D}, \Omega_{n}\right)$ are holomorphic maps, and $\zeta_{j} \in \mathbb{D}$ such that $\tilde{\varphi}_{j}(0)=B, \tilde{\varphi}_{j}\left(\zeta_{j}\right)$ $=A_{j}, \lim _{j \rightarrow \infty}\left|\zeta_{j}\right|=\ell$. Consider $\varphi_{j}:=\sigma \circ \tilde{\varphi}_{j}$, and (by Montel's Theorem) extract a subsequence converging to $\varphi \in \mathcal{O}\left(\mathbb{D}, \mathbb{G}_{n}\right)$ and such that $\zeta_{j} \rightarrow \zeta_{0}$.

By the necessary condition in Proposition 4.1, $\Theta_{B}\left(\varphi_{j}\right)=0, \varphi_{j}(0)=0$ and $\varphi_{j}\left(\zeta_{j}\right)=\sigma\left(A_{j}\right)$. So compact convergence implies that $\varphi(0)=0, \varphi\left(\zeta_{0}\right)=\sigma(A)$ and $\Theta_{B}(\varphi)=0$. By the sufficiency part of Proposition 4.1, there exists $\tilde{\varphi} \in \mathcal{O}\left(\mathbb{D}, \Omega_{n}\right)$ such that $\tilde{\varphi}(0)=B, \tilde{\varphi}\left(\zeta_{0}\right)=A$. Therefore $l_{\Omega_{n}}(A, B) \leq\left|\zeta_{0}\right|=\ell$.

4.2. Proof of Proposition 4.1, It will be enough to find a map $\tilde{\varphi}$ that satisfies the conclusion only with $\tilde{\varphi}(0)=B^{\prime}$ and $\tilde{\varphi}\left(\zeta_{0}\right)=A^{\prime}$, where $B^{\prime}, A^{\prime}$ are conjugate to $B, A$, respectively, as in [1, Proof of Theorem 2.1]. Furthermore, any cyclic matrix with the same spectrum as $A$ will be conjugate to $A$. So it is enough to check that $\tilde{\varphi}(0)=B$, where $B$ is in Jordan form, and $\tilde{\varphi}(\zeta)$ is cyclic for $\zeta \neq 0$.

We use the following notations: $B=\left(b_{i, j}\right)_{1 \leq i, j \leq n}$. For two integers $k \leq l$, $[k . l]:=\{i \in \mathbb{Z}: k \leq i \leq l\}$. Let $r$ stand for the rank of $B$. Write

$$
F_{0}:=\left\{j: b_{i, j}=0 \text { for } 1 \leq i \leq n\right\}:=\left\{1=b_{1}<b_{2}<\cdots<b_{n-r}\right\} .
$$


For $j \in F_{1}:=[1 . . n] \backslash F_{0}, b_{j-1, j}=1, b_{i, j}=0$ for $i \neq j-1$. We can choose the Jordan form so that $b_{l+1}-b_{l}$ is increasing for $1 \leq l \leq n-r$, with the convention $b_{n-r+1}:=n+1$.

Let us give the differential conditions satisfied by a map $\sigma \circ \tilde{\varphi}$ at 0 . To do so, we must study the homogeneity of the functions $\sigma_{i}(B+M)$ in terms of the entries of $M$. Let $M=\left(m_{k, l}\right)_{1 \leq k, l \leq n}$. For $E \subset\{1, \ldots, n\}$, denote $M_{E}:=\left(m_{k, l}\right)_{k, l \in E}$.

$$
\begin{aligned}
\sigma_{i}(B+M) & =\sum_{E \subset\{1, \ldots, n\}, \# E=i} \operatorname{det}(B+M)_{E} \\
& =\sum_{E \subset\{1, \ldots, n\}, \# E=i} \sum_{\theta \in \mathcal{S}(E)}(-1)^{\operatorname{sgn}(\theta)} \prod_{e \in E}\left(b_{e, \theta(e)}+m_{e, \theta(e)}\right),
\end{aligned}
$$

where $\mathcal{S}(E)$ stands for the permutation group of $E$. This is a polynomial of degree $\leq i$ in the entries of $M$.

Lemma 4.2. Let $d_{i}:=1+\#\left(F_{0} \cap[(n-i+2) . . n]\right)$. The lowest order terms of $\sigma_{i}(B+M)$ are of degree $d_{i}$ (in the entries of $M$ ).

Proof. The terms in the polynomial in (4.1) are of the form

$$
\pm \prod_{e \in E_{1}} b_{e, \theta(e)} \cdot \prod_{e \in E_{2}} m_{e, \theta(e)},
$$

where $E=E_{1} \cup E_{2}, E_{1} \cap E_{2}=\emptyset$. Non zero terms of degree $\# E_{2}$ have sets $E_{1}$ such that

$$
b_{e, \theta(e)}=1 \quad \forall e \in E_{1},
$$

so $\theta(e)=e+1$ and $e+1 \in F_{1}$.

Write $J_{k}:=\left[\left(b_{k}+1\right) . .\left(b_{k+1}-1\right)\right]$, so that $F_{1}=\bigcup_{k} J_{k}$ (some of those may be empty), and $E_{1, k}:=\left\{e \in E_{1}: e+1 \in J_{k}\right\}$. If $E_{1, k} \neq \emptyset$, then $1+\max E_{1, k}=$ $\theta\left(\max E_{1, k}\right) \in E$, and since it cannot be in any of the $J_{l}$, it must belong to $E_{2}$. Thus

$$
d:=\# E_{2} \geq \#\left\{k: E_{1, k} \neq \emptyset\right\} .
$$

We use the partition $E=E_{2} \cup \bigcup_{k} E_{1, k}$ :

$$
\begin{aligned}
\# E=i=d+\sum_{k: E_{1, k} \neq \emptyset} \# E_{1, k} \leq d+\sum_{k: E_{1, k} \neq \emptyset} \# J_{k} \\
\quad \leq d+\sum_{k=n-r-d+1}^{k=n-r}\left(b_{k+1}-b_{k}-1\right)=(n+1)-b_{n-r-d+1} .
\end{aligned}
$$

Therefore $b_{n-r-d+1} \leq(n+1)-i<n-i+2$, which means that $[(n-i+2) . . n]$ contains no more elements of $F_{0}$ than those greater or equal to $b_{n-r-d+2}$, of which there are $d-1$. So $\#\left(F_{0} \cap[(n-i+2) . . n]\right) \leq d-1$; i.e. $\# E_{2} \geq d_{i}$.

To show that this bound is attained, choose

$$
E_{1}:=\left\{e \in[(n-i+1) . .(n-1)]: e+1 \in F_{1}\right\} .
$$

Then $\# E_{1}=i-d_{i}$, and we can define the permutation $\theta$ by $\theta(e)=e+1, n-i+1 \leq$ $e \leq n$, and $\theta(n)=n-i+1$, so that $E=[(n-i+1) . . n]$ and $\# E=i$, as required.

Corollary 4.3. If $\varphi=\left(\varphi_{1}, \ldots, \varphi_{n}\right)=\sigma \circ \tilde{\varphi}$ with $\tilde{\varphi} \in \mathcal{O}\left(\mathbb{D}, \Omega_{n}\right)$, $\tilde{\varphi}(0)=B$, then $\varphi_{i}^{(k)}(0)=0,0 \leq k \leq d_{i}-1$. 
This gives additional (differential) conditions whenever $d_{i} \geq 2$.

Conversely, given a map $\varphi \in \mathcal{O}\left(\mathbb{D}, \mathbb{G}_{n}\right)$ satisfying the conclusion of Corollary 4.3, let

$$
\psi(\zeta):=\left(\begin{array}{ccccc}
0 & f_{2} & 0 & \cdots & 0 \\
0 & 0 & \ddots & & \vdots \\
\vdots & & \ddots & f_{n-1} & 0 \\
0 & 0 & \cdots & 0 & f_{n} \\
\psi_{n} & \psi_{n-1} & \cdots & \psi_{2} & \psi_{1}
\end{array}\right) .
$$

Lemma 4.4. For a matrix $\psi$ as above, $\sigma_{i}(\psi)=(-1)^{i+1} \psi_{i} \prod_{k=n-i+2}^{n} f_{k}$.

Proof. Expanding with respect to the last row,

$$
\operatorname{det}\left(X I_{n}-M\right)=X^{n}+\sum_{j=1}^{n}(-1)^{j+n}\left(-\psi_{n+1-j}\right) X^{j-1} \prod_{k=j+1}^{n}\left(-f_{k}\right),
$$

and we find the coefficient of $(-1)^{i} X^{n-i}$ by setting $j=n-i+1$.

We define a lifting by taking $\psi$ with $f_{j}(\zeta)=1$ for $j \in F_{1}, f_{j}(\zeta)=\zeta$ for $j \in F_{0}$, $\psi_{j}(\zeta)=(-1)^{i+1} \zeta^{-d_{j}+1} \varphi_{j}(\zeta)$. Those coefficients are holomorphic, and $\psi(0)=B$, since $\varphi$ satisfies the conclusion of Corollary 4.3 Lemma 4.4 shows that $\sigma \circ \psi=\varphi$. Finally, for any $\zeta \neq 0$, the matrix $\psi(\zeta)$ is cyclic, and $\sigma \circ \psi\left(\zeta_{0}\right)=\sigma(A)$, so we can modify $\psi$ as indicated at the beginning of the section, to obtain a lifting through A.

4.3. The cases $n=2$ or 3 . The only cases to deal with are those where $B$ is derogatory (non cyclic). As above, we can reduce ourselves to the case where one eigenvalue of $B$ is 0 .

When $n=2$, the only derogatory matrices are scalar; therefore we only need to consider the case $B=0$ and Proposition 4.1 (or indeed, [1, Theorem 2.8]) settles the question. When $n=3$ and $B$ is derogatory, either it has a single eigenvalue, or it has two distinct eigenvalues, one of which has an eigenspace of dimension 2 . We then may assume that $\operatorname{dim} \operatorname{ker} B=2$, and, say,

$$
B=\left(\begin{array}{ccc}
0 & 0 & 0 \\
0 & 0 & 0 \\
0 & 0 & \lambda_{1}
\end{array}\right)
$$

for some $\lambda_{1} \in \mathbb{D} \backslash\{0\}$.

If $\varphi=\sigma \circ \tilde{\varphi}$, simple determinant calculations show that

$$
\varphi(0)=\sigma(B)=\left(\lambda_{1}, 0,0\right), \text { and } \varphi_{3}^{\prime}(0)=0 .
$$

Assume we have a map $\varphi \in \mathcal{O}\left(\mathbb{D}, \mathbb{G}_{3}\right)$ such that (4.3) holds. Set

$$
\psi(\zeta):=\left(\begin{array}{ccc}
0 & \zeta & 0 \\
0 & 0 & \zeta \\
\zeta^{-2} \varphi_{3}(\zeta) & -\zeta^{-1} \varphi_{2}(\zeta) & \varphi_{1}(\zeta)
\end{array}\right)
$$

Then $\sigma \circ \psi=\varphi, \psi(0)$ is conjugate to $B$ (not necessarily equal to it), and for any $\zeta \neq 0, e_{3}$ is a cyclic vector for $\psi(\zeta)$. 


\section{An example}

Recall from [11, Proposition 4] that when $A=t I$ and $B$ is cyclic, the function $l_{\Omega_{n}}(., B)$ is continuous at $A$, or equivalently the function $l_{\Omega_{n}}$ is continuous at $(A, B)$, if and only if all the eigenvalues of $B$ are equal. For $n=2$, this covers all the derogatory cases. By using an automorphism of $\Omega_{n}$, the situation quickly reduces to the case $t=0$. The next example in the case $n=3$ is then

$$
A:=\left(\begin{array}{ccc}
0 & 0 & 0 \\
0 & 0 & 1 \\
0 & 0 & 0
\end{array}\right)
$$

Example 5.1. Taking

$$
B=\left(\begin{array}{ccc}
\varepsilon & 0 & 0 \\
0 & j \varepsilon & 0 \\
0 & 0 & j^{2} \varepsilon
\end{array}\right), \text { where } \varepsilon>0, j=-1 / 2+i \sqrt{3} / 2
$$

and $\varepsilon$ is small enough, the function $l_{\Omega_{3}}(., B)$ is discontinuous at $A$.

Indeed, we clearly have $\operatorname{sp} A=\{0\}, \sigma(A)=(0,0,0) ; B$ is non-derogatory. The eigenvalues of $B$ are $\varepsilon, j \varepsilon$ and $j^{2} \varepsilon$. Thus $\sigma(B)=\left(0,0, \varepsilon^{3}\right)$.

We find $r>0$ such that $\mathbb{B}(0, r) \subset \mathbb{G}_{3}$, where $\mathbb{B}(0, r)$ denotes the Euclidean ball with center at 0 and radius $r$. For $\varepsilon$ chosen small enough, $\sigma(B) \in \mathbb{B}(0, r)$. By the definition of the Lempert function and [8, Proposition 3.1.10], we conclude that

$$
l_{\mathbb{G}_{3}}(\sigma(A), \sigma(B))=l_{\mathbb{G}_{3}}(0, \sigma(B)) \leq l_{\mathbb{B}(0, r)}(0, \sigma(B))=\frac{\|\sigma(B)\|}{r}=\frac{\varepsilon^{3}}{r} .
$$

On the other hand, if there is an analytic function $\varphi: \mathbb{D} \rightarrow \Omega_{3}$ such that $\varphi(0)=A$ and $\varphi(\zeta)=B$, then by (3.1) we have $\varepsilon^{2}=\max \left\{\varepsilon^{2}, \varepsilon^{3}\right\} \leq|\zeta|$. It follows that

$$
l_{\Omega_{3}}(A, B) \geq \varepsilon^{2}>\frac{\varepsilon^{3}}{r} \geq l_{\mathbb{G}_{3}}(\sigma(A), \sigma(B))
$$

for $\varepsilon$ small enough.

Example 5.2. If the eigenvalues of $B$ are equal, then the function $l_{\Omega_{3}}(., B)$ is continuous at $A$ (moreover $l_{\Omega_{3}}$ is continuous at $(A, B)$ ).

Indeed, if the eigenvalues of $B$ are equal, say to $\mu$, then, by [11] and (1.1) we have

$$
|\mu| \geq l_{\Omega_{3}}(A, B) \geq l_{\mathbb{G}_{3}}(\sigma(A), \sigma(B)) .
$$

On the other hand, if $C_{\mathbb{G}_{3}}$ is the Carathéodory pseudodistance of $\mathbb{G}_{3}$, then

$$
l_{\mathbb{G}_{3}}(\sigma(A), \sigma(B)) \geq C_{\mathbb{G}_{3}}(\sigma(A), \sigma(B)) \geq \sup _{|\lambda|=1}\left|\frac{f_{\lambda}(\sigma(B))-f_{\lambda}(\sigma(A))}{1-\overline{f_{\lambda}(\sigma(A))} f_{\lambda}(\sigma(B))}\right|=|\mu|,
$$

where $f_{\lambda}(S)=\frac{s_{1}+2 s_{2} \lambda+3 s_{3} \lambda^{2}}{3+2 s_{1} \lambda+s_{2} \lambda^{2}}, \forall S=\left(s_{1}, s_{2}, s_{3}\right) \in \mathbb{G}_{3}, \lambda \in \overline{\mathbb{D}}$ (for the last inequality see [4] or [9]). Thus $l_{\Omega_{3}}(A, B)=l_{\mathbb{G}_{3}}(\sigma(A), \sigma(B))=|\mu|$. 


\section{The PluRicomplex GReEN AND Lempert FunCtions ARE NOT EQUAL}

Let $D$ be a domain in $\mathbb{C}^{n}$.

Definition 6.1. For $(a, z) \in D \times D$, the pluricomplex Green function with pole at $a$, evaluated at $z$ is

$$
\begin{aligned}
g_{D}(a, z):=\sup \{u(z): u: D \rightarrow & {[0,1), \log u \in P S H(D), } \\
& \exists C=C(u, a)>0, \forall w \in D: u(w) \leq C\|w-a\|\}
\end{aligned}
$$

where $P S H(D)$ denotes the family of all functions plurisubharmonic on $D$ (and \| \| is the Euclidean norm in $\left.\mathbb{C}^{n}\right)$.

Note that what we call the Green function is the exponential of the usual Green function with logarithmic singularity.

The formulas for the Carathéodory and the Lempert functions on $\mathbb{G}_{2}$ were obtained by Agler and Young [2]. Using the fact that $C_{\mathbb{G}_{2}}=l_{\mathbb{G}_{2}}$, Costara [5] has obtained a formula for the Carathéodory and the Lempert functions on $\Omega_{2}$. He proved that on $\Omega_{2}$ the Carathéodory and the Lempert functions do not coincide. The same holds for the Green function, for any $n$.

Proposition 6.2. Let $A$ be a cyclic matrix in $\Omega_{n}$ such that at least two of the eigenvalues of $A$ are not equal. Then there exists a matrix $B \in \Omega_{n}$ such that

$$
l_{\Omega_{n}}(A, B)>g_{\Omega_{n}}(A, B) .
$$

For Green functions with several poles, there are cases of strict inequality even for the usual bidisk [12.

Proof. We need the following.

Proposition 6.3 (Edigarian-Zwonek). Let $A, B \in \Omega_{n}$. Then

$$
g_{\Omega_{n}}(A, B)=g_{\Omega_{n}}\left(A, \operatorname{diag}\left(\mu_{1}, \mu_{2}, \cdots, \mu_{n}\right)\right)
$$

where $\operatorname{sp}(B)=\left\{\mu_{j}, 1 \leq j \leq n\right\}$, with the eigenvalues repeated according to multiplicity.

Take $B_{\alpha}=\left(b_{i, j}\right)_{1 \leq i, j \leq n} \in \Omega_{n}$, where $b_{1,1}=b_{2,2}=\cdots=b_{n, n}=\mu \in \mathbb{D} ; b_{j-1, j}=$ $\alpha \in \mathbb{C}, \forall 2 \leq j \leq n$, and all other coefficients $b_{i, j}=0,1 \leq i, j \leq n$.

Since $B_{0}$ is a scalar matrix, by using [10], we have discontinuity of the Lempert function $l_{\Omega_{n}}$ at $\left(A, B_{0}\right)$. Then by Proposition 1.2 ,

$$
l_{\Omega_{n}}\left(A, B_{0}\right)>l_{G_{n}}\left(\sigma(A), \sigma\left(B_{0}\right)\right) .
$$

Now consider $\alpha \neq 0$. Then $A, B_{\alpha}$ are cyclic matrices. It implies that

$$
l_{G_{n}}\left(\sigma(A), \sigma\left(B_{0}\right)\right)=l_{G_{n}}\left(\sigma(A), \sigma\left(B_{\alpha}\right)\right)=l_{\Omega_{n}}\left(A, B_{\alpha}\right) .
$$

On the other hand,

$$
l_{\Omega_{n}}\left(A, B_{\alpha}\right) \geq g_{\Omega_{n}}\left(A, B_{\alpha}\right)=g_{\Omega_{n}}\left(A, B_{0}\right),
$$

where the last equality follows from (6.1).

Thus $l_{\Omega_{n}}\left(A, B_{0}\right)>g_{\Omega_{n}}\left(A, B_{0}\right)$. 


\section{REFERENCES}

1. J. Agler, N. J. Young, The two-point spectral Nevanlinna-Pick problem, Integral Equations and Operator Theory 37 (2000), 375-385. MR1780117 (2001g:47025)

2. J. Agler, N. J. Young, The hyperbolic geometry of the symmetrized bidisc, J. Geom. Anal. 14 (2004) 375-403. MR2077158 (2005e:32022)

3. G. Bharali, Some new observations on interpolation in the spectral unit ball, Integral Equations Operator Theory 59 (2007), no. 3, 329-343. MR2363012 (2009a:47025)

4. C. Costara, On the spectral Nevanlinna-Pick problem, Studia Math. Soc. 170 (2005), 23-55. MR2142182 (2006d:30054)

5. C. Costara, The $2 \times 2$ spectral Nevanlinna-Pick problem, J. London Math. Soc. (2) 71 (2005) 684-702. MR2132378 (2006b:30063)

6. A. Edigarian, W. Zwonek, Geometry of the symmetrized polydisc, Arch. Math. (Basel) 84 (2005), 364-374. MR2135687 (2006b:32020)

7. R. A. Horn, C. R. Johnson, Matrix Analysis, Cambridge University Press, Cambridge, New York, Melbourne, 1985. MR832183(87e:15001)

8. M. Jarnicki, P. Pflug, Invariant Distances and Metrics in Complex Analysis, de Gruyter Exp. Math., 9, de Gruyter, Berlin, New York, 1993. MR.1242120(94k:32039)

9. N. Nikolov, P. Pflug, W. Zwonek, The Lempert function of the symmetrized polydisc in higher dimension is not a distance, Proc. Amer. Math. Soc. 135 (2007), no. 9, 2921-2928. MR 2317970 (2008e:32017)

10. N. Nikolov, P. J. Thomas, On the zero set of the Kobayashi-Royden pseudometric of the spectral unit ball, Ann. Pol. Math. 93 (2008), no. 1, 53-68 MR2383341 (2008k:32028)

11. N. Nikolov, P. J. Thomas, W. Zwonek, Discontinuity of the Lempert function and the Kobayashi-Royden metric of the spectral ball, Integral Equations Operator Theory 61 (2008), no. 3, 401-412. MR2417504 (2009c:32023)

12. P. J. Thomas, N. V. Trao, Pluricomplex Green and Lempert functions for equally weighted poles, Ark. Mat. 41 (2003), no. 2, 381-400. MR2011928 (2004m:32067)

Institut de Mathématiques de Toulouse, Université de Toulouse, UPS, INSA, UT1, UTM, F-31062 Toulouse, France

E-mail address: pthomas@math.univ-toulouse.fr

Department of Mathematics, Hanoi National University of Education, 136 Xuan Thuy str - Cau Giay, Hanoi, Vietnam

E-mail address: ngvtrao@yahoo.com 